International Mathematical Forum, 2, 2007, no. 37, 1811 - 1830

\title{
A Generalized Like-distance in Convex Programming
}

\author{
Romulo A. Castillo ${ }^{1}$ \\ Department of Mathematics \\ Universidad Centroccidental "Lisandro Alvarado" \\ Barquisimeto, Venezuela \\ romulo@uicm.ucla.edu.ve \\ Eibar Hernandez ${ }^{2}$ \\ Department of Mathematics \\ Universidad Centroccidental "Lisandro Alvarado" \\ Barquisimeto, Venezuela \\ ehernand@ucla.edu.ve \\ Jorge Campos \\ Section of Mathematics, UNEXPO \\ Barquisimeto, Venezuela \\ jcampos@bqto.unexpo.edu.ve
}

\begin{abstract}
We consider a generalized like-distance which contains as degenerate cases $\phi$-divergences and like-distances with second order homogeneous kernel. The motivation to get this like-distance comes from studying shifted penalty functions in the primal space. These penalty functions do not necessarily have to pass through the origin with slope one and their conjugate functions allow negative values. For a particular case we get a generalization of the Kullback-Liebler entropy distance. This like-distance can be seen as the difference between a sequence of Bregman distances and their linear approximations for specific values of the arguments. Dual and primal convergence results are shown, particularly, we show that each limit point of the sequence generated by the proximal method defined by the generalized like-distance applied to the dual problem is an optimal dual solution.
\end{abstract}

\footnotetext{
${ }^{1}$ This author was partially supported by CDCHT-UCLA-Venezuela.

${ }^{2}$ This author was partially supported by DFPA-UCLA-Venezuela.
} 
Mathematics Subject Classification: 90C99, 90C25, 49M30

Keywords: Proximal point methods, convex programming, multiplier methods

\section{Introduction}

We consider the convex programming problem defined by

$$
(P) \quad f^{*}=\inf \left\{f_{0}(x): f_{i}(x) \leq 0 \quad i=1, \ldots, m\right\}
$$

where $f_{i}: \mathbb{R}^{n} \rightarrow \mathbb{R}$ for $i=0,1, \ldots, m$ are closed proper convex functions. The associated dual convex problem can be written by

$$
\text { (D) } \quad d^{*}=\inf \{-d(\mu): \mu \geq 0\}
$$

where $d(\mu)=\inf \left\{l(x, \mu): x \in \mathbb{R}^{n}\right\}$ and

$$
l(x, \mu)=f_{0}(x)+\sum_{i=1}^{m} \mu_{i} f_{i}(x)
$$

is the Lagrangian function.

We assume the following hypothesis:

(H1) The optimal set for problem $(P)$ is nonempty and compact.

(H2) There exist $\bar{x}$ such that $f_{i}(\bar{x})<0$ for $i=1, \ldots, m$.

The problem $(P)$ can be solved using different approaches, for example, we can use augmented Lagrangian methods which can be introduced from a primal view point, see [3], [14],[2] or from a dual view point where the multiplier method is constructed applying Fenchel's theory of duality to the proximal point method, see [17], [9], [6], [1].

Proximal point methods with $\phi$ divergences to solve $(D)$, generate a sequence $\left\{\mu^{k}\right\} \in \mathbb{R}_{++}^{m}$ such that $\mu^{0} \in \mathbb{R}_{++}^{m}$ and

$$
\mu^{k+1}=\operatorname{argmin}_{\mu \geq 0}\left\{-d(\mu)+r^{k} d_{\phi}\left(\mu, \mu_{k}\right)\right\}
$$

where

$$
\begin{gathered}
0<\underline{r}<r^{k}<\bar{r} \leq 1 \\
d_{\phi}: \mathbb{R}_{+}^{m} \times \mathbb{R}_{++}^{m} \rightarrow \mathbb{R} \text { is given by } d_{\phi}(s, \mu)=\sum_{i=1}^{m} \mu_{i} \phi\left(\frac{s_{i}}{\mu_{i}}\right)
\end{gathered}
$$

and $\phi$ is a nonnegative and strictly convex function that passes by the point $(1,0)$ and it is well studied in [9]. Other like-distances have been used in 
different contexts with different properties and results, for example in [2], the parameter $r$ was proposed as a function of the multiplier $\mu$ and when $r(\mu)=\mu$ we get the like-distance studied in [1] which is homogeneous of order two and given by the expression

$$
d_{\phi}(s, \mu)=\sum_{i=1}^{m} \mu_{i}^{2} \phi\left(\frac{s_{i}}{\mu_{i}}\right) .
$$

Proximal point methods with Bregman distances solve $(D)$ by using iterates in (3) with $D_{h}$ instead of $d_{\phi}$ and given by

$$
D_{h}(s, \mu)=h(s)-h(\mu)-\nabla h_{\mu}(\mu)^{t}(s-\mu)
$$

where $h$ is a Bregman function, see [12]. In section 2 we consider a new likedistance to solve problem $(D)$ which has interesting properties and is given by the expression

$$
d_{\theta^{*}}^{p}(s, \mu)=\sum_{i=1}^{m}\left[\frac{\mu_{i}^{p}}{c} \theta^{*}\left(\frac{c s_{i}}{\mu_{i}}\right)-\frac{\mu_{i}^{p}}{c} \theta^{*}(c)-\mu_{i}^{p-1}\left(\theta^{*}\right)^{\prime}(c)\left(s_{i}-\mu_{i}\right)\right]
$$

where $\theta^{*}$ is the conjugate function of the penalty function $\theta$ which does not necessarily pass through the origin and satisfies $\theta^{\prime}(0)=\kappa, \kappa>0, p \geq 0$ and $c \in \mathbb{R}_{++}$satisfies $\left(\theta^{*}\right)^{\prime}(c)=\tilde{y} \in \mathbb{R}$.

For $\mu \in \mathbb{R}_{++}^{m}$ fixed, if we define $h_{\mu}: \mathbb{R}_{+}^{m} \rightarrow \mathbb{R}$ by $h_{\mu}(s)=\sum_{i=1}^{m} \frac{\mu_{i}^{p}}{c} \theta^{*}\left(\frac{c s_{i}}{\mu_{i}}\right)$ we get $d_{\theta^{*}}^{p}(s, \mu)=h_{\mu}(s)-h_{\mu}(\mu)-\nabla h_{\mu}(\mu)^{t}(s-\mu)$, i.e., $d_{\theta^{*}}^{p}$ can be seen as the difference between $h_{\mu}(s)$ and its linear approximation at $s=\mu$. We observe that $\theta^{*}$ is a translation of the function $\phi$ used in $\phi$-divergences, in this case, with minimal point at $\left(\kappa, \theta^{*}(\kappa)\right)$ and $\theta^{*}(\kappa) \in \mathbb{R}$. In section 3 we show dual and primal results based on a proximal method and an augmented Lagrangian algorithm. Sections 4 and 5 and the appendix present the concluding remarks. We get the like-distance by applying the conjugacity theory to a shifted penalty function used in an augmented Lagrangian approach, so we consider first a family of penalty functions and we show how the like-distance defined in (7) naturally arises.

\section{Getting the like-distance}

In order to get the generalized like-distance in a natural way we study first a family of penalty functions in the context of multiplier methods.

\subsection{Penalty functions}

Let $\theta$ be a strictly increasing twice differentiable strictly convex function defined on $(-\infty, b), 0<b \leq+\infty$, such that: 
1a) $0<\theta^{\prime}(0)=\kappa<+\infty$.

2a) $\lim _{t \rightarrow b} \theta^{\prime}(t)=+\infty$.

3a) $\lim _{t \rightarrow-\infty} \theta^{\prime}(t)=0$.

4a) $\theta^{\prime \prime}(t) \geq \frac{1}{M}, \quad \forall t \in[0, b]$ and for some $M>0$.

Condition 1a) has already been considered in Bregman distances approach, see [12],[5] but not in like-distances ones, see [9],[1], [14], [2], in our case the penalty function does not need to pass through the origin with slope one. Associated with the function $\theta$ we consider and denote its conjugate function $\theta^{*}$, see [15] which satisfies the following properties:

1b) $\theta^{*}$ is a strictly convex differentiable function on $(0,+\infty)$.

2b) $\theta^{*}$ is decreasing on $(0, \kappa)$ and increasing on $(\kappa,+\infty)$ with $\theta^{*}(\kappa) \in \mathbb{R}$.

3b) $\lim _{t \rightarrow 0^{+}}\left(\theta^{*}\right)^{\prime}(t)=-\infty$ and $\lim _{t \rightarrow+\infty}\left(\theta^{*}\right)^{\prime}(t)=+\infty$.

4b) $\left(\theta^{*}\right)^{\prime \prime}(t) \leq M$ for all $t \geq \kappa=\theta^{\prime}(0)$.

Condition $2 \mathrm{~b}$ ) with $\kappa=1$ is used in all the like-distances known until now. Observe that negative values for $\theta^{*}$ are allowed.

\section{Example 2.1.}

For $\theta(t)=e^{t+1}$, we have $\theta(0)=e, \theta^{\prime}(0)=e=\kappa,\left(\theta^{*}\right)^{\prime}(e)=0$ and $\theta^{*}(e)=-e$. So, $\theta^{*}$ has its minimal point at $(e,-e)$. We show the graphics of $\theta$ and $\theta^{*}$ in the next figure.
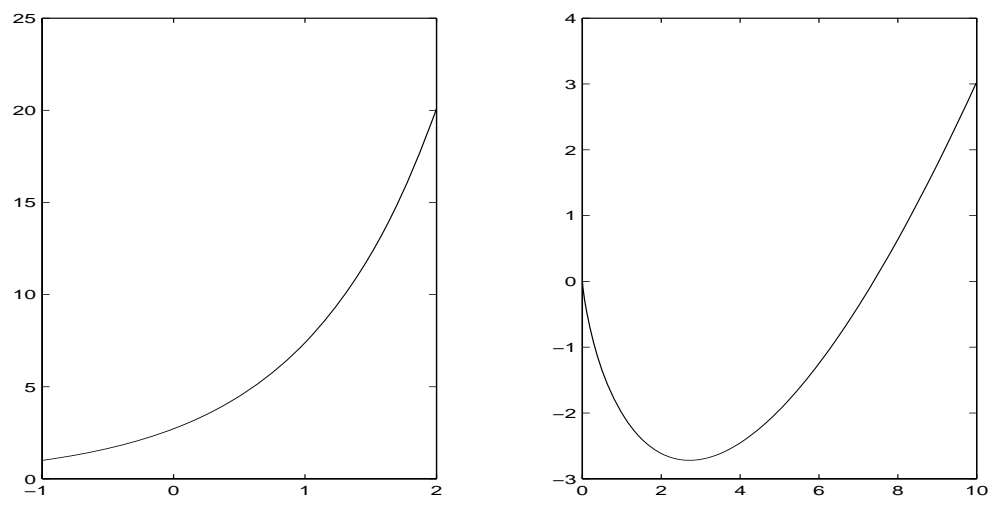

Fig.1 Graphics of $\theta$ and $\theta^{*}$

\subsection{Shift in penalty functions}

We use a constant shift to define a generalized penalty function which will be used in section (3.2) in the context of the multiplier's method.

We choose $\tilde{y} \in \mathbb{R}$ so that $\theta^{\prime}(\tilde{y})=c$ with $c \in \mathbb{R}_{++}$. According to the corollary 23.5.1 in [15] we have

$$
\left(\theta^{\prime}\right)^{-1}=\left(\theta^{*}\right)^{\prime}
$$


and so

$$
\theta^{\prime}(\tilde{y})=c \Leftrightarrow \tilde{y}=\left(\theta^{*}\right)^{\prime}(c) .
$$

Given $p \geq 0, r \in(0,1]$, we define the generalized penalty function with shift $P_{p}$ as:

$$
y \in \mathbb{R}^{m}, \mu \in \mathbb{R}_{++}^{m} \mapsto P_{p}(y, \mu, r, c)=\sum_{i=1}^{m} P_{p, i}\left(y_{i}, \mu_{i}, r, c\right)
$$

where $P_{p, i}\left(y_{i}, \mu_{i}, r, c\right)=r \frac{\mu_{i}^{p}}{c}\left[\theta\left(\frac{y_{i}}{\mu_{i}^{p-1} r}+\tilde{y}\right)-\theta(\tilde{y})\right]$ for $i=1, \ldots, m, \theta$ satisfies conditions 1a)-4a) and $\theta^{\prime}(\tilde{y})=c$.

Note that $P_{p, i}\left(0, \mu_{i}, r, c\right)=0$ for $i=1, \ldots, m$ and

$$
\left(P_{p, i}\right)_{1}^{\prime}\left(y_{i}, \mu_{i}, r, c\right)=\frac{\mu_{i}}{c} \theta^{\prime}\left(\frac{y_{i}}{\mu_{i}^{p-1} r}+\tilde{y}\right),
$$

where $\left(P_{p, i}\right)_{1}^{\prime}=\frac{\partial P_{p, i}}{\partial y_{i}}$, so by $(9)$

$$
\left(P_{p, i}\right)_{1}^{\prime}\left(0, \mu_{i}, r, c\right)=\frac{\mu_{i}}{c} \theta^{\prime}(\tilde{y})=\mu_{i}, \text { for } i=1, \ldots, m
$$

\section{Remark}

An interesting fact in the penalty function $P_{p}$ is that it considers for the first time rational exponents on the multiplier vectors, although we show only convergence results for $p \geq 2$.

Geometrically the shift is a translation that satisfies the equation (11). The conditions $\theta(0)=0$ and $\theta^{\prime}(0)=1$ were used in all the multiplier methods known until now that lead to like-distances, see [9], [1], [2], [14], but not in this one, what really matter is that equation (11) holds.

Another relevant aspect of the shift in this penalty function is that it allows us to construct a generalized like-distance in the dual space applying the conjugacity theory as shown in the next proposition.

Proposition 2.2. Let $\theta$ be a penalty function satisfying conditions 1a)-4a). Given $p \geq 0, r \in(0,1], \tilde{y} \in \mathbb{R}, c \in \mathbb{R}_{++}$, consider

$$
y \in \mathbb{R}^{m}, \mu \in \mathbb{R}_{++}^{m} \mapsto P_{p}(y, \mu, r, c)=\sum_{i=1}^{m} r \frac{\mu_{i}^{p}}{c}\left[\theta\left(\frac{y_{i}}{\mu_{i}^{p-1} r}+\tilde{y}\right)-\theta(\tilde{y})\right]
$$

where $\theta^{\prime}(\tilde{y})=c$ then

$$
P_{p, \mu, r, c}^{*}(s)=r \sum_{i=1}^{m}\left[\frac{\mu_{i}^{p}}{c} \theta^{*}\left(\frac{c s_{i}}{\mu_{i}}\right)-\frac{\mu_{i}^{p}}{c} \theta^{*}(c)-\mu_{i}^{p-1}\left(\theta^{*}\right)^{\prime}(c)\left(s_{i}-\mu_{i}\right)\right] .
$$


Proof.

Consider

$$
P_{p, i}\left(y_{i}, \mu_{i}, r, c\right)=\frac{r}{c} \mu_{i}^{p}\left[\theta\left(\frac{y_{i}}{\mu_{i}^{p-1} r}+\tilde{y}\right)-\theta(\tilde{y})\right] \quad \text { for } i=1, \ldots, m
$$

where $\theta$ satisfies conditions 1a)-4a) and $\theta^{\prime}(\tilde{y})=c$.

Let $r, c, \mu_{i}$, for $i=1, \ldots, m$ be fixed and proposition 1.3.1 in [11], we have

$$
\begin{aligned}
P_{p, \mu_{i}, r, c}^{*}\left(s_{i}\right) & =\left[\frac{r}{c} \mu_{i}^{p} \theta\left(\frac{y_{i}}{\mu_{i}^{p-1} r}+\tilde{y}\right)-\frac{r}{c} \mu_{i}^{p} \theta(\tilde{y})\right]^{*} \quad \text { for } i=1, \ldots, m \\
& =\left[\frac{r}{c} \mu_{i}^{p} \theta\left(\frac{y_{i}}{\mu_{i}^{p-1} r} \tilde{y}+\tilde{y}\right)\right]^{*}+\frac{r}{c} \mu_{i}^{p} \theta(\tilde{y}) \\
& =\frac{r}{c} \mu_{i}^{p} \theta^{*}\left(\frac{c s_{i}}{\mu_{i}}\right)-r \mu_{i}^{p-1} s_{i} \tilde{y}+\frac{r}{c} \mu_{i}^{p} \theta(\tilde{y}) .
\end{aligned}
$$

By theorem 25.3 in [15], if $\tilde{y} \in \operatorname{dom} \theta$ and $c \in \operatorname{dom} \theta^{*}$ we know that $\theta(\tilde{y})+\theta^{*}(c)=c \tilde{y}$ for $i=1, \ldots, m$, by using (9) we have

$$
\begin{aligned}
P_{p, \mu_{i}, r, c}^{*}\left(s_{i}\right) & =\frac{r}{c} \mu_{i}^{p} \theta^{*}\left(\frac{c s_{i}}{\mu_{i}}\right)-\mu_{i}^{p-1} r s_{i} \tilde{y}+\frac{r}{c} \mu_{i}^{p}\left[c \tilde{y}-\theta^{*}(c)\right. \\
& =\frac{r}{c} \mu_{i}^{p} \theta^{*}\left(\frac{c s_{i}}{\mu_{i}}\right)-\frac{r}{c} \mu_{i}^{p} \theta^{*}(c)-\mu_{i}^{p-1} r \tilde{y}\left(s_{i}-\mu_{i}\right) \\
& =\frac{r}{c} \mu_{i}^{p} \theta^{*}\left(\frac{c s_{i}}{\mu_{i}}\right)-\frac{r}{c} \mu_{i}^{p} \theta^{*}(c)-\mu_{i}^{p-1} r\left(\theta^{*}\right)^{\prime}(c)\left(s_{i}-\mu_{i}\right) .
\end{aligned}
$$

Finally,

$$
P_{p, \mu, r, c}^{*}(s)=r \sum_{i=1}^{m}\left[\frac{\mu_{i}^{p}}{c} \theta^{*}\left(\frac{c s_{i}}{\mu_{i}}\right)-\frac{\mu_{i}^{p}}{c} \theta^{*}(c)-\mu_{i}^{p-1}\left(\theta^{*}\right)^{\prime}(c)\left(s_{i}-\mu_{i}\right)\right] \text {. }
$$

\subsection{The generalized like-distance}

According with definition 2.1 in [10], given $S \subset \mathbb{R}^{n} ; \quad d: S \times S \rightarrow \mathbb{R}$ is called a divergence measure in $S$ if and only if:

i) $d(x, y) \geq 0, \quad \forall x, y \in S$.

ii) If $\left\{x^{k}\right\} \subset S, x \in S$, then $\lim _{k \rightarrow+\infty} d\left(x, x^{k}\right)=0 \Leftrightarrow \lim _{k \rightarrow+\infty} x^{k}=x$.

iii) The partial level sets $\Gamma_{1}(y, \nu)=\{x \in S: d(x, y) \leq \nu\}$ are bounded $\forall y \in S$ and $\forall \nu>0$.

iv) The partial level sets $\Gamma_{2}(x, \nu)=\{y \in S: d(x, y) \leq \nu\}$ are bounded $\forall x \in S$ and $\forall \nu>0$.

Using (13), we define the generalized like-distance as

$$
d_{\theta^{*}}^{p}(x, y)=\sum_{i=1}^{m}\left[\frac{y_{i}^{p}}{c} \theta^{*}\left(\frac{c x_{i}}{y_{i}}\right)-\frac{y_{i}^{p}}{c} \theta^{*}(c)-y_{i}^{p-1}\left(\theta^{*}\right)^{\prime}(c)\left(x_{i}-y_{i}\right)\right] .
$$

In the appendix we show that it is a divergent measure. Observe that $d_{\theta^{*}}^{p}(\cdot, y)$ is a strictly convex function because $\theta^{*}$ is one. 
Note that for $p=1, c=1, \tilde{y}=0$ with $\theta(\tilde{y})=0$ we get:

$$
P_{1}^{*}(s, \mu, r, c)=r d_{\theta^{*}}(s, \mu)=r \sum_{i=1}^{m} \mu_{i} \theta^{*}\left(\frac{s_{i}}{\mu_{i}}\right)
$$

which is used in proximal methods with $\phi$-divergences, see [10], [14]. For $p=2, c=1, \tilde{y}=0$ with $\theta(\tilde{y})=0$ we get

$$
P_{2}^{*}(s, \mu, r, c)=r \tilde{d}_{\theta^{*}}(s, \mu)=r \sum_{i=1}^{m} \mu_{i}^{2} \theta^{*}\left(\frac{s_{i}}{\mu_{i}}\right)
$$

the second order homogeneous kernel used in [1] and in [2] for a specific case.

\section{Example 2.3.}

For $m=1$ and $\theta(t)=e^{t}$, we have $\theta^{*}(s)=s \ln (s)-s,\left(\theta^{*}\right)^{\prime}(s)=\ln (s)$ and $d_{\theta^{*}}^{p}(s, \mu)=\mu^{p-1}\left(s \ln \left(\frac{s}{\mu}\right)-s+\mu\right)$. This like-distance can be considered as a generalization of the Kullback-Leibler entropy distance. The same expression can be obtained from $\theta(t)=e^{t}-1$ or from $\theta(t)=e^{t+K}$ with $K \in \mathbb{R}$.

\subsubsection{Geometric Interpretation}

Consider the generalized like-distance defined in (14) and given $\mu \in \mathbb{R}_{++}^{m}$, we define the convex function

$$
h_{\mu}: \mathbb{R}_{+}^{m} \rightarrow \mathbb{R} \quad \text { as } h_{\mu}(s)=\sum_{i=1}^{m} \frac{\mu_{i}^{p}}{c} \theta^{*}\left(\frac{c s_{i}}{\mu_{i}}\right) .
$$

Note that $h_{\mu}(\mu)=\sum_{i=1}^{m} \frac{\mu_{i}^{p}}{c} \theta^{*}(c)$ and

$\nabla h_{\mu}(s)=\left(\mu_{1}^{p-1}\left(\theta^{*}\right)^{\prime}\left(\frac{c s_{1}}{\mu_{1}}\right), \ldots, \mu_{m}^{p-1}\left(\theta^{*}\right)^{\prime}\left(\frac{c s_{m}}{\mu_{m}}\right)\right)^{t}$

so that $\nabla h_{\mu}(\mu)=\left(\mu_{1}^{p-1} \theta^{* \prime}(c), \ldots, \mu_{m}^{p-1} \theta^{* \prime}(c)\right)^{t}$.

We have then

$$
d_{\theta^{*}}^{p}(s, \mu)=h_{\mu}(s)-h_{\mu}(\mu)-\nabla h_{\mu}(\mu)^{t}(s-\mu),
$$

that is, for each $\mu, d_{\theta^{*}}^{p}(s, \mu)$ is the difference between $h_{\mu}(s)$ and its linear approximation at $s=\mu$.

It can be observed that for each $\mu>0, h_{\mu}(s)=\sum_{i=1}^{m} \frac{\mu_{i}^{p}}{c} \theta^{*}\left(\frac{c s_{i}}{\mu_{i}}\right)$ is a strictly 
convex function and it is not difficult to prove that, for each $\mu>0$ it generates a Bregman distance $D_{h_{\mu}}: \mathbb{R}_{+}^{m} \times \mathbb{R}_{++}^{m} \rightarrow \mathbb{R}$ given by

$$
D_{h_{\mu}}(x, y)=h_{\mu}(x)-h_{\mu}(y)-\nabla h_{\mu}(y)^{t}(x-y),
$$

see [12], so we have for each $\mu>0$, the generalized like-distance satisfies

$$
d_{\theta^{*}}^{p}(s, \mu)=h_{\mu}(s)-h_{\mu}(\mu)-\nabla h_{\mu}(\mu)^{t}(s-\mu)=D_{h_{\mu}}(s, \mu) .
$$

If we consider a sequence $\left\{\mu^{k}\right\}$ in (15) and in (16) we have in (15) a sequence of Bregman distances, so that the generalized like-distance at $\mu=\mu^{k}$ coincide with $D_{h^{k}}(x, y)$ at $y=\mu^{k}$. This suggest the posibility of studying proximal methods with a sequence of Bregman distances depending on a parameter or varying the induced Bregman function at each iteration. This will not be considered in this paper.

Figure 2 below shows the graph of $h_{\mu}(s)$ and its linear approximation at $\mu$ for $\mu=2, \frac{3}{2}, 1, c=\frac{1}{2}, p=\frac{5}{2}$ and $\theta^{*}$ as in example 1 . On the right, the graph of $d_{\theta^{*}}^{p}(s, \mu)$ for the same values.
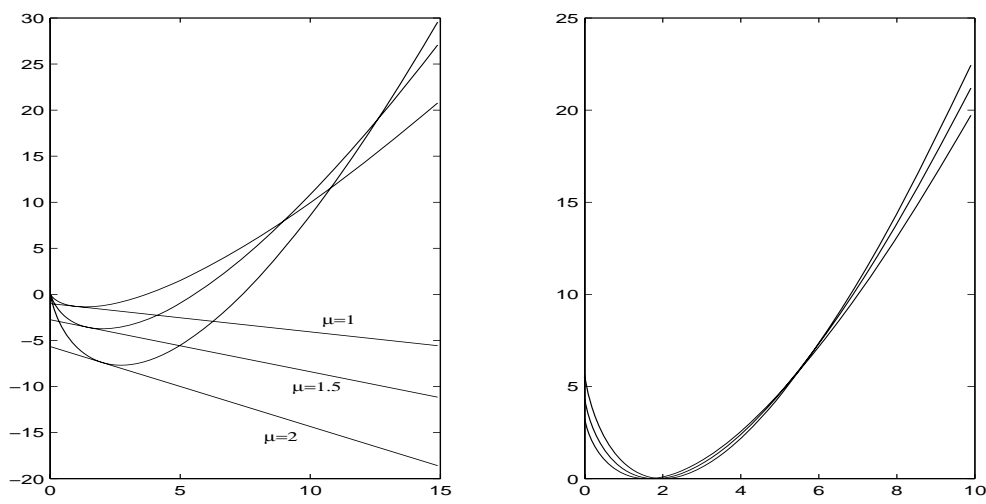

Fig.2

\section{Methods and theorems}

\subsection{Proximal method}

We consider the generalized like-distance applied directly over the dual problem. The proximal method to solve problem $(D)$ defined in $(1)$ generates a sequence $\left\{\mu^{k}\right\}$ such that $\mu^{0} \in \mathbb{R}_{++}^{m}$ and

$$
\mu^{k+1}=\operatorname{argmin}\left\{-d(\mu)+r^{k} d_{\theta^{*}}^{p}\left(\mu, \mu^{k}\right)\right\}
$$

where $r^{k} \in[\widehat{r}, \bar{r}] \subset(0,+\infty)$ and $\theta^{*}$ as defined in section $(2.1)$.

By optimality condition we have

$$
0 \in \partial\left[-d\left(\mu^{k+1}\right)+r^{k} d_{\theta^{*}}^{p}\left(\mu^{k+1}, \mu^{k}\right)\right]
$$


or equivalently

$$
-r^{k}\left(\left(\mu_{1}^{k}\right)^{p-1}\left[\theta^{* \prime}\left(\frac{c \mu_{1}^{k+1}}{\mu_{1}^{k}}\right)-\theta^{* \prime}(c)\right], \ldots,\left(\mu_{m}^{k}\right)^{p-1}\left[\theta^{* \prime}\left(\frac{c \mu_{m}^{k+1}}{\mu_{m}^{k}}\right)-\theta^{* \prime}(c)\right]\right) \in \partial\left(-d\left(\mu^{k+1}\right)\right) .
$$

Proposition 3.1. $\left\{-d\left(\mu^{k}\right)\right\}$ is a non-increasing convergent sequence.

Proof. Due to the optimality conditions in (17) we have

$\mu^{k+1}=\operatorname{argmin}_{\mu>0}\left\{-d(\mu)+r^{k} d_{\theta^{*}}^{p}\left(\mu, \mu^{k}\right)\right\}$, so $-d\left(\mu^{k+1}\right)+r^{k} d_{\theta^{*}}^{p}\left(\mu^{k+1}, \mu^{k}\right) \leq$ $-d\left(\mu^{k}\right)+r^{k} d_{\theta^{*}}^{p}\left(\mu^{k}, \mu^{k}\right)=-d\left(\mu^{k}\right)$, that is $-d\left(\mu^{k+1}\right) \leq-d\left(\mu^{k}\right)$. By duality theory, $f^{*}$ is a lower bound for $\left\{-d\left(\mu^{k}\right)\right\}$, so $\left\{-d\left(\mu^{k}\right)\right\}$ is convergent.

Proposition 3.2. The sequence $\left\{\mu^{k}\right\}$ generated by (17) is bounded.

Proof. By (H2) and since $-d$ is a proper convex function, the level set $\Lambda=\left\{\mu \in \mathbb{R}_{+}^{m} /-d(\mu) \leq-d\left(\mu_{0}\right)\right\}$ is compact and by proposition 3.1, $\mu^{k} \in \Lambda$ for all $k$, so $\mu^{k}$ is bounded.

In the next subsection, based on proposition 2.2 a primal multiplier method associated to the proximal one will be obtained using the generalized penalty functions defined in (10).

\subsection{Augmented Lagrangian algorithm}

Consider problem $(P)$ with hypothesis $(\mathrm{H} 1)$ and $(\mathrm{H} 2)$ and the $\theta$-functions satisfying conditions 1a)-4a) in section (2.1).

Given $p \geq 0, r \in(0,1], \tilde{y} \in \mathbb{R}, c \in \mathbb{R}_{++}$with $\theta^{\prime}(\tilde{y})=c$, the augmented Lagrangian function is given by

$$
L_{r, c}(x, \mu)=f_{0}(x)+\sum_{i=1}^{m} P_{p, i}\left(f_{i}(x), \mu_{i}, r, c\right)
$$

where $P_{p, i}\left(f_{i}(x), \mu_{i}, r, c\right)=\frac{\mu_{i}^{p}}{c}\left[\theta\left(\frac{f_{i}(x)}{\mu_{i}^{p-1} r}+\tilde{y}\right)-\theta(\tilde{y})\right]$ for $i=1, \cdots, m$.

The multiplier method associated to the proximal one is given by:

$$
\begin{gathered}
x^{k+1} \in \operatorname{argmin}\left\{f_{0}(x)+\sum_{i=1}^{m} P_{p, i}\left(f_{i}(x), \mu_{i}^{k}, r^{k}, c\right)\right\} \\
\mu_{i}^{k+1}=\frac{\mu_{i}^{k}}{c} \theta^{\prime}\left(\frac{f_{i}\left(x^{k+1}\right)}{\left(\mu_{i}^{k}\right)^{p-1} r^{k}}+\tilde{y}\right) \quad \text { for } i=1, \cdots, m \text { and } r^{k} \in(0,1] .
\end{gathered}
$$


Observe that

$$
0 \in \partial_{x} L_{r^{k}, c}\left(x^{k+1}, \mu^{k}\right) \Leftrightarrow 0 \in \partial_{x} l\left(x^{k+1}, \mu^{k+1}\right)
$$

where $l$ is the lagrangian function defined in (2).

The next proposition shows that the sequences defined in (20) and (17) are the same.

Proposition 3.3. Let $\left\{\widehat{\mu}^{k}\right\}$ be the sequence generated by (17) which solves the dual problem $(D)$ and let $\left\{x^{k}\right\}$ and $\left\{\mu^{k}\right\}$ be the sequences generated by (19) and (20) which solve the primal problem $(P)$. If $\mu^{0}=\widehat{\mu}^{0}$, then $\forall k>0: \mu^{k}=\widehat{\mu}^{k}$.

The proof is direct from theorem 7.1 in [8].

\subsection{Convergence results}

This section is inspired from the convergence theorems in [2] and [14].

From (14) we can write

$$
d_{\theta^{*}}^{p}(s, \mu)=\sum_{i=1}^{m} d\left(s_{i}, \mu_{i}\right)
$$

where $d\left(s_{i}, \mu_{i}\right)=\frac{\mu_{i}^{p}}{c} \theta^{*}\left(\frac{c s_{i}}{\mu_{i}}\right)-\frac{\mu_{i}^{p}}{c} \theta^{*}(c)-\mu_{i}^{p-1}\left(\theta^{*}\right)^{\prime}(c)\left(s_{i}-\mu_{i}\right)$ for $i=\ldots, m$ and

$d_{1}^{\prime}\left(s_{i}, \mu_{i}\right)=\frac{\partial d\left(s_{i}, \mu_{i}\right)}{\partial s_{i}}=\mu_{i}^{p-1}\left[\left(\theta^{*}\right)^{\prime}\left(\frac{c s_{i}}{\mu_{i}}\right)-\left(\theta^{*}\right)^{\prime}(c)\right]$.

Lemma 3.4. Let $s, \mu$ be positive real numbers with $s>\mu$ then

$$
d(s, \mu) \geq \frac{1}{2} \frac{\left[d_{1}^{\prime}(s, \mu)\right]^{2}}{c M \mu^{p-2}}
$$

where $M=\max \left\{\left(\theta^{*}\right)^{\prime \prime}(t) / t \geq \kappa=\theta^{\prime}(0)\right\}$ and $c=\theta^{\prime}(\tilde{y})$.

Proof. Consider the quadratic function

$$
q(t)=q(s)+(t-s) d_{1}^{\prime}(s, \mu)+\frac{1}{2}(t-s)^{2} c M \mu^{p-2}
$$

So

SO

$$
\begin{aligned}
q^{\prime}(t) & =d_{1}^{\prime}(s, \mu)+(t-s) c M \mu^{p-2} \text { and } \\
q^{\prime}(t)=0 & \Leftrightarrow d_{1}^{\prime}(s, \mu)+t c M \mu^{p-2}-s c M \mu^{p-2}=0 \\
& \Leftrightarrow t=\frac{-d_{1}^{\prime}(s, \mu)}{c M \mu^{p-2}}+\frac{s c M \mu^{p-2}}{c M \mu^{p-2}}=\frac{-d_{1}^{\prime}(s, \mu)}{c M \mu^{p-2}}+s
\end{aligned}
$$

$$
t^{*}=\frac{-d_{1}^{\prime}(s, \mu)}{c M \mu^{p-2}}+s
$$


is a minimizer of $q(\cdot)$.

Since $s>\mu>0$

$$
d_{1}^{\prime}(s, \mu)=\mu^{p-1}\left[\left(\theta^{*}\right)^{\prime}\left(\frac{c s}{\mu}\right)-\left(\theta^{*}\right)^{\prime}(c)\right]>0 .
$$

Then, from $(22) t^{*}<s$.

Furthermore,

$$
\begin{aligned}
d_{1}^{\prime}(s, \mu) & =d_{1}^{\prime}(s, \mu)-d_{1}^{\prime}(\mu, \mu)=(s-\mu) d_{1}^{\prime \prime}(\hat{\mu}, \mu) \text { for some } \hat{\mu} \in[\mu, s] \\
& =(s-\mu) \mu^{p-2}\left(\theta^{*}\right)^{\prime \prime}\left(\frac{c \hat{\mu}}{\mu}\right) c \leq(s-\mu) \mu^{p-2} c M .
\end{aligned}
$$

So $\frac{d_{1}^{\prime}(s, \mu)}{c M \mu^{p-2}} \leq s-\mu$ and

$$
\mu \leq s-\frac{d_{1}^{\prime}(s, \mu)}{c M \mu^{p-2}}=t^{*}
$$

then $\mu \leq t^{*}<s$.

On the other hand, for all $t \in[\mu, s)$

$$
\begin{aligned}
d_{1}^{\prime}(s, \mu)-d_{1}^{\prime}(t, \mu) & =(s-t) d_{1}^{\prime \prime}(\bar{t}, \mu) \text { for some } t \leq \bar{t} \leq s \\
& =(s-t) \mu^{p-2}\left(\theta^{*}\right)^{\prime \prime}\left(\frac{c \bar{t}}{\mu}\right) c \\
& \leq(s-t) \mu^{p-2} c M
\end{aligned}
$$

so $d_{1}^{\prime}(s, \mu) \leq d_{1}^{\prime}(t, \mu)+(s-t) \mu^{p-2} c M$ for all $\mu \leq t \leq s$

and

$$
d_{1}^{\prime}(t, \mu) \geq d_{1}^{\prime}(s, \mu)+(t-s) \mu^{p-2} c M=q^{\prime}(t) .
$$

We have then

$$
d_{1}^{\prime}(t, \mu) \geq q^{\prime}(t) \text { for all } \mu \leq t \leq s .
$$

Since $\mu \leq t^{*}<s$, integrating from $t^{*}$ to $s$ we get

$$
d(s, \mu)-d\left(t^{*}, \mu\right) \geq q(s)-q\left(t^{*}\right) .
$$

So $d(s, \mu) \geq q(s)-q\left(t^{*}\right)+d\left(t^{*}, \mu\right) \geq q(s)-q\left(t^{*}\right)$ and

$$
d(s, \mu) \geq q(s)-q(s)-\left(t^{*}-s\right) d_{1}^{\prime}(s, \mu)-\frac{1}{2}\left(t^{*}-s\right)^{2} c M \mu^{p-2} .
$$

From $(23) t^{*}-s=\frac{-d_{1}^{\prime}(s, \mu)}{c M \mu^{p-2}}$,

replacing in (24) we have

$$
\begin{gathered}
d(s, \mu) \geq \frac{\left[d_{1}^{\prime}(s, \mu)\right]^{2}}{c M \mu^{p-2}}-\frac{1}{2} \frac{\left[d_{1}^{\prime}(s, \mu)\right]^{2}}{c M \mu^{p-2}} \\
=\frac{1}{2} \frac{\left[d_{1}^{\prime}(s, \mu)\right]^{2}}{c M \mu^{p-2}} .
\end{gathered}
$$


Finally,

$$
d(s, \mu) \geq \frac{1}{2} \frac{\left[d_{1}^{\prime}(s, \mu)\right]^{2}}{c M \mu^{p-2}}
$$

The next proposition uses implicitly a similar version of property (D4) in [2], and it is valid for $p \geq 2$.

Proposition 3.5. (Asymptotic feasibility) Consider $p \geq 2$. The sequence $\left\{\left[f_{i}\left(x^{k}\right)\right]_{+}\right\} \quad$ converges to 0 for all $i=1, \ldots, m$ where $[y]_{+}=\max \{0, y\}$.

Proof. Let us suppose by absurd that $\left\{\left[f_{i}\left(x^{k}\right)\right]\right\} \nrightarrow 0$, then there exists $\left\{l_{k}\right\}$ such that $l_{k} \rightarrow+\infty$ with $l_{k}<l_{k+1}$ and $\exists \epsilon>0$ such that $f_{i_{0}}\left(x^{l_{k}}\right)>\epsilon$ for some $i_{0} \in\{1, \ldots, m\}$.

We have $d(\mu)=\inf \left\{f_{0}(x)+\sum_{i=1}^{m} \mu_{i} f_{i}(x)\right\} \leq f_{0}\left(x^{k+1}\right)+\sum_{i=1}^{m} \mu_{i} f_{i}\left(x^{k+1}\right)$

$$
\begin{aligned}
& =f_{0}\left(x^{k+1}\right)+\sum_{i=1}^{m} \mu_{i}^{k+1} f_{i}\left(x^{k+1}\right)+\sum_{i=1}^{m}\left(\mu_{i}-\mu_{i}^{k+1}\right) f_{i}\left(x^{k+1}\right) \\
& =d\left(\mu_{i}^{k+1}\right)+\sum_{i=1}^{m}\left(\mu_{i}-\mu_{i}^{k+1}\right) f_{i}\left(x^{k+1}\right) \text { for } i=1, \ldots, m .
\end{aligned}
$$

From (20),

$$
\mu_{i}^{k+1}=\frac{\mu_{i}^{k}}{c} \theta^{\prime}\left(\frac{f_{i}\left(x^{k+1}\right)}{\left(\mu_{i}^{k}\right)^{p-1} r^{k}}+\tilde{y}\right) \quad \text { for } i=1, \ldots, m
$$

and by using $\left(\theta^{\prime}\right)^{-1}=\left(\theta^{*}\right)^{\prime}$ we get

$$
r^{k}\left(\mu_{i}^{k}\right)^{p-1}\left[\left(\theta^{*}\right)^{\prime}\left(\frac{c \mu_{i}^{k+1}}{\mu_{i}^{k}}\right)-\left(\theta^{*}\right)^{\prime}(c)\right]=f_{i}\left(x^{k+1}\right) \quad \text { for } i=1, \ldots, m .
$$

Using notation in (21),

$$
d\left(s_{i}, \mu_{i}\right)=\frac{\mu_{i}^{p}}{c} \theta^{*}\left(\frac{c s_{i}}{\mu_{i}}\right)-\frac{\mu_{i}^{p}}{c} \theta^{*}(c)-\mu_{i}^{p-1}\left(\theta^{*}\right)^{\prime}(c)\left(s_{i}-\mu_{i}\right) \quad \text { for } i=1, \ldots, m
$$

and

$$
d_{1}^{\prime}\left(s_{i}, \mu_{i}\right)=\mu_{i}^{p-1}\left[\left(\theta^{*}\right)^{\prime}\left(\frac{c s_{i}}{\mu_{i}}\right)-\left(\theta^{*}\right)^{\prime}(c)\right] \quad \text { for } i=1, \ldots, m,
$$

from (26) and (27)

$$
\frac{f_{i}\left(x^{k+1}\right)}{r^{k}}=d_{1}^{\prime}\left(\mu_{i}^{k+1}, \mu_{i}^{k}\right) \quad \text { for } i=1, \ldots, m .
$$


Since $f_{i_{0}}\left(x^{l_{k+1}}\right)>\epsilon$, then $r^{k} d_{1}^{\prime}\left(\mu_{i_{0}}^{l_{k+1}}, \mu_{i_{0}}^{l_{k}}\right)>\epsilon>0$.

This implies $d_{1}^{\prime}\left(\mu_{i_{0}}^{l_{k+1}}, \mu_{i_{0}}^{l_{k}}\right)>0$ and so $\mu_{i_{0}}^{l_{k+1}}>\mu_{i_{0}}^{l_{k}}$, and we can use proposition 3.4 .

Since $\mu^{k+1}=\operatorname{argmin}\left\{-d(\mu)+d_{\theta^{*}}^{p}\left(\mu, \mu^{k}\right)\right\}$, we have $-d\left(\mu^{k+1}\right)+d_{\theta^{*}}^{p}\left(\mu^{k+1}, \mu^{k}\right) \leq$ $-d\left(\mu^{k}\right)+d_{\theta^{*}}^{p}\left(\mu^{k}, \mu^{k}\right)=-d\left(\mu^{k}\right)$,

so $d\left(\mu_{k+1}\right)-d\left(\mu_{k}\right) \geq d_{\theta^{*}}^{p}\left(\mu^{k+1}, \mu^{k}\right) \geq 0$.

Then $d\left(\mu^{l_{k+1}}\right)-d\left(\mu^{l_{k}}\right) \geq d_{\theta^{*}}^{p}\left(\mu^{l_{k+1}}, \mu^{l_{k}}\right)=\sum_{i=1}^{m} d\left(\mu_{i}^{l_{k+1}}, \mu_{i}^{l_{k}}\right) \geq d\left(\mu_{i_{0}}^{l_{k+1}}, \mu_{i_{0}}^{l_{k}}\right)$.

By proposition 3.2, let $\widehat{\mu}$ an upper bound of $\left\{\mu^{k}\right\}$ and by lemma 3.4 we have

$$
d\left(\mu^{l_{k+1}}\right)-d\left(\mu^{l_{k}}\right) \geq \frac{1}{2 c M} \frac{\left[d_{1}^{\prime}\left(\mu_{i_{0}}^{l_{k+1}}, \mu_{i_{0}}^{l_{k}}\right)\right]^{2}}{\left(\mu_{i_{0}}^{l_{k}}\right)^{p-2}} \geq \frac{\epsilon^{2}}{2 c M \widehat{\mu}_{i_{0}}^{p-2}}=\delta>0
$$

for $p \geq 2$.

Then $d\left(\mu^{l_{k+1}}\right)-d\left(\mu^{l_{k}}\right) \geq \delta>0$ and taking limits when $k \rightarrow \infty$ we have that 0 is greater than a positive value, which is a contradiction.

Finally, $\lim _{k \rightarrow+\infty}\left[f_{i}\left(x^{k}\right)\right]_{+}=0$.

The next proposition use the following affirmation:

Fact 1: For all $t, t \frac{\theta^{\prime}(t+\tilde{y})}{c} \geq t$.

$$
\text { Proof. If } t>0 \Rightarrow t+\widetilde{y}>\widetilde{y} \Rightarrow \theta^{\prime}(t+\widetilde{y})>\theta^{\prime}(\widetilde{y})=c
$$

$\Rightarrow \frac{\theta^{\prime}(t+\widetilde{y})}{c}>1 \Rightarrow t \frac{\theta^{\prime}(t+\widetilde{y})}{c}>t$.

If $t<0 \Rightarrow t+\widetilde{y}<\widetilde{y}^{c} \Rightarrow \theta^{\prime}(t+\widetilde{y})<\theta^{\prime}(\widetilde{y})=c$

$\Rightarrow \frac{\theta^{\prime}(t+\widetilde{y})}{c}<1 \Rightarrow t \frac{\theta^{\prime}(t+\widetilde{y})}{c}>t$.

Proposition 3.6. Let $\left\{x^{k}\right\}$ and $\left\{\mu^{k}\right\}$ be the sequences generated by (19) and (20), then $\lim _{k \rightarrow+\infty} \mu_{i}^{k} f_{i}\left(x^{k}\right)=0$ for $i=1, \cdots, m$.

Proof. By absurd, suppose there exists $i_{0} \in\{1, \ldots, m\}, \epsilon>0$ and an infinite index set $\left\{k_{j}\right\}$ such that

$$
\left|\mu_{i_{0}}^{k_{j}+1} f_{i_{0}}\left(x^{k_{j}+1}\right)\right| \geq \epsilon \text { for all } \mathrm{j} .
$$

Since $\left\{\mu^{k}\right\}$ is bounded, there exists $\widehat{\mu}>0$ such that:

$$
0<\mu^{k} \leq \widehat{\mu} \quad \text { for all } \mathrm{k}
$$

hence

$$
\left|\mu_{i_{0}}^{k_{j}+1} f_{i_{0}}\left(x^{k_{j}+1}\right)\right| \geq \epsilon \Rightarrow\left|f_{i_{0}}\left(x^{k_{j}+1}\right)\right| \geq \frac{\epsilon}{\widehat{\mu}_{i_{0}}}
$$


But $\left\{\left[f_{i}\left(x^{k}\right)\right]_{+}\right\}$converges to 0 , for all $i=1, \ldots, m$ hence $f_{i_{0}}\left(x^{k_{j}+1}\right) \geq \frac{\epsilon}{\hat{\mu}_{i_{0}}}$ is true only for a finite set of index $k_{j}$, so we can consider without lost of generality

$$
f_{i_{0}}\left(x^{k_{j}+1}\right) \leq-\frac{\epsilon}{\widehat{\mu}_{i_{0}}}
$$

for all $j$.

Since $\left(f_{1}\left(x^{k+1}\right), \ldots, f_{m}\left(x^{k+1}\right)\right)^{t} \in \partial d\left(\mu^{k+1}\right)$ and $d$ is a concave function, then

$$
\sum_{i=1}^{m} f_{i}\left(x^{k_{j}+1}\right)\left(\mu_{i}^{k_{j}+1}-\mu_{i}^{k_{j}}\right) \leq d\left(\mu^{k_{j}+1}\right)-d\left(\mu^{k_{j}}\right)
$$

Since $\mu_{i}^{k+1}=\frac{\mu_{i}^{k}}{c} \theta^{\prime}\left(\frac{f_{i}\left(x^{k+1}\right)}{\left(\mu_{i}^{k}\right)^{p-1} r^{k}}+\widetilde{y}\right)$ for $i=1, \ldots, m$ we have

$$
\begin{array}{r}
f_{i}\left(x^{k_{j}+1}\right)\left(\mu_{i}^{k_{j}+1}-\mu_{i}^{k_{j}}\right)=\mu_{i}^{k_{j}} f_{i}\left(x^{k_{j}+1}\right)\left(\frac{\mu_{i}^{k_{j}+1}}{\mu_{i}^{k_{j}}}-1\right) \\
=\mu_{i}^{k_{j}} f_{i}\left(x^{k_{j}+1}\right)\left(\frac{1}{c} \theta^{\prime}\left(\frac{f_{i}\left(x^{k_{j}+1}\right)}{\left(\mu_{i}^{k_{j}}\right)^{p-1} r^{k}}+\widetilde{y}\right)-1\right) \\
=\frac{\mu_{i}^{k_{j}} f_{i}\left(x^{k_{j}+1}\right)}{c} \theta^{\prime}\left(\frac{f_{i}\left(x^{k_{j}+1}\right)}{\left(\mu_{i}^{k_{j}}\right)^{p-1} r^{k}}+\widetilde{y}\right)-\mu_{i}^{k_{j}} f_{i}\left(x^{k_{j}+1}\right) .
\end{array}
$$

Since $\theta$ is a strictly convex function, $\theta^{\prime \prime}(t)>0$ for all $t \in(-\infty, b)$, then $\theta^{\prime}$ is increasing and so using (33) and fact 1

$$
\begin{aligned}
f_{i}\left(x^{k_{j}+1}\right)\left(\mu_{i}^{\left.k_{j}+1\right)}-\mu_{i}^{k_{j}}\right) & =\frac{r^{k}\left(\mu_{i}^{k_{j}}\right)^{p}}{c}\left[\frac{f_{i}\left(x^{k_{j}+1}\right)}{\left(\mu_{i}^{k_{j}}\right)^{p-1} r^{k}} \theta^{\prime}\left(\frac{f_{i}\left(x^{k_{j}+1}\right)}{\left(\mu_{i}^{k_{j}}\right)^{p-1} r^{k}}+\widetilde{y}\right)\right]-\mu_{i}^{k_{j}} f_{i}\left(x^{k_{j}+1}\right) \\
& \geq \mu_{i}^{k_{j}} f_{i}\left(x^{k_{j}+1}\right)-\mu_{i}^{k_{j}} f_{i}\left(x^{k_{j}+1}\right) \\
& =0
\end{aligned}
$$

hence $0 \leq \sum_{i=1}^{m} f_{i}\left(x^{k_{j}+1}\right)\left(\mu_{i}^{k_{j}+1}-\mu_{i}^{k_{j}}\right) \leq d\left(\mu^{k_{j}+1}\right)-d\left(\mu^{k_{j}}\right)$,

since $\left\{d\left(\mu^{k}\right)\right\}$ is convergent, then $\lim _{j \rightarrow+\infty}\left[d\left(\mu^{k_{j}+1}\right)-d\left(\mu^{k_{j}}\right)\right]=0$

and so

$\lim _{j \rightarrow+\infty} \sum_{i=1}^{m} f_{i}\left(x^{k_{j}+1}\right)\left(\mu_{i}^{k_{j}+1}-\mu_{i}^{k_{j}}\right)=0$.

Since $f_{i}\left(x^{k_{j}+1}\right)\left(\mu_{i}^{k_{j}+1}-\mu_{i}^{k_{j}}\right) \geq 0$ for all $i=1, \ldots, m$

we have $\lim _{j \rightarrow+\infty} f_{i}\left(x^{k_{j}+1}\right)\left(\mu_{i}^{k_{j}+1}-\mu_{i}^{k_{j}}\right)=0$ for all $i=1, \ldots, m$.

So from (33),

$$
\lim _{j \rightarrow+\infty} \mu_{i}^{k_{j}} f_{i}\left(x^{k_{j}+1}\right)\left[\frac{1}{c} \theta^{\prime}\left(\frac{f_{i}\left(x^{k_{j}+1}\right)}{\left(\mu_{i}^{k_{j}}\right)^{p-1} r^{k}}+\widetilde{y}\right)-1\right]=0 \text { for all } i=1, \ldots, m .
$$


On the other hand

$$
\frac{f_{i_{0}}\left(x^{k_{j}+1}\right)}{r^{k}\left(\mu_{i_{0}}^{k_{j}}\right)^{p-1}}+\widetilde{y} \leq \frac{-\epsilon}{r^{k}\left(\mu_{i_{0}}^{k_{j}}\right)^{p-1} \widehat{\mu_{i_{0}}}}+\widetilde{y}<0+\widetilde{y}
$$

and since $\theta^{\prime}$ is increasing,

$$
\theta^{\prime}\left(\frac{f_{i_{0}}\left(x^{k_{j}+1}\right)}{r^{k}\left(\mu_{i_{0}}^{k_{j}}\right)^{p-1}}+\widetilde{y}\right) \leq \theta^{\prime}\left(\frac{-\epsilon}{r^{k}\left(\mu_{i_{0}}^{k_{j}}\right)^{p-1} \widehat{\mu_{i_{0}}}}+\widetilde{y}\right)<\theta^{\prime}(\widetilde{y})=c
$$

SO

$$
\frac{1}{c} \theta^{\prime}\left(\frac{f_{i_{0}}\left(x^{k_{j}+1}\right)}{r^{k}\left(\mu_{i_{0}}^{k_{j}}\right)^{p-1}}+\widetilde{y}\right) \leq \frac{1}{c} \theta^{\prime}\left(\frac{-\epsilon}{r^{k}\left(\mu_{i_{0}}^{k_{j}}\right)^{p-1} \widehat{\mu_{i_{0}}}}+\widetilde{y}\right)<1 .
$$

From (34) and (35)

$$
\lim _{j \rightarrow+\infty} \mu_{i_{0}}^{k_{j}} f_{i_{0}}\left(x^{k_{j}+1}\right)=0
$$

Since

$$
\lim _{j \rightarrow+\infty} f_{i}\left(x^{k_{j}+1}\right)\left(\mu_{i}^{k_{j}+1}-\mu_{i}^{k_{j}}\right)=0
$$

then $\lim _{j \rightarrow+\infty} \mu_{i_{0}}^{k_{j}+1} f_{i_{0}}\left(x^{k_{j}+1}\right)=0$, which is a contradiction.

Consequently

$$
\mu_{i}^{k} f_{i}\left(x^{k}\right) \rightarrow 0
$$

for all $i=1, \ldots, m$

Theorem 3.7. The sequence $\left\{f_{0}\left(x^{k}\right)\right\}$ converges to $\widehat{f}$, each limit point of the sequences $\left\{x^{k}\right\}$ and $\left\{\mu^{k}\right\}$ generated by the method (19) and (20) (or (19) and (17)) are optimal solutions for the problems $(P)$ and $(D)$ respectively.

Proof. Since $\left\{x^{k}\right\}$ is asymptotically feasible, for all $\epsilon>0, f_{0}\left(x^{k}\right) \geq \widehat{f}-\epsilon$ for sufficiently large $k$.

According to $(\mathrm{H} 2)$, the optimal set of problem $(D)$ is nonempty and compact, $\widehat{f}=\widehat{d}$ where

$$
\widehat{f}=\min \left\{f_{0}(x): x \in R^{n}, f_{i}(x) \leq 0, i=1, \ldots, m\right\}
$$

and

$$
\widehat{d}=\sup \left\{d(\mu): \mu \in R_{+}^{m}\right\}
$$


Furthermore, for each $\beta<\widehat{d}$ the level set $\left\{\mu \in R_{+}^{m}: d(\mu) \geq \beta\right\}$ is compact. But

$$
\widehat{f}=\widehat{d} \geq d\left(\mu^{k}\right)=\inf _{x}\left\{l\left(x, \mu^{k}\right)\right\}=l\left(x^{k}, \mu^{k}\right)=f_{0}\left(x^{k}\right)+\sum_{i=1}^{m} \mu_{i}^{k} f_{i}\left(x^{k}\right)
$$

and according to proposition $3.6\left\{\mu_{i}^{k} f_{i}\left(x^{k}\right)\right\}$ converges to 0 for all $i=1, \ldots, m$ so

for all $\epsilon>0, \widehat{f}-\epsilon \leq f_{0}\left(x^{k}\right) \leq \widehat{f}-\sum_{i=1}^{m} \mu_{i}^{k} f_{i}\left(x^{k}\right)<\widehat{f}+\epsilon$ for sufficiently large $k$ and so

$$
f_{0}\left(x^{k}\right) \rightarrow \widehat{f}
$$

By proposition 3.5, for all $\epsilon>0$ and for $i=1, \ldots, m, f_{i}\left(x^{k}\right) \leq \epsilon$ for sufficiently large $k$, then $f_{0}\left(x^{k}\right) \leq \widehat{f}+\epsilon$ and $f_{i}\left(x^{k}\right) \leq \epsilon$ for $i=1, \ldots, m$ and sufficiently large $k$. According to (H1) and corollary 20 in [7] the set

$$
\left\{x \in R^{n}: f_{i}(x) \leq \alpha, f_{0}(x) \leq \beta\right\}
$$

is compact for any $\alpha, \beta$, then $\left\{x^{k}\right\}$ is bounded and by the proposition 3.2 the sequence $\left\{\mu^{k}\right\}$ also is bounded. If $\bar{x}$ is a limit point of $\left\{x^{k}\right\}$ then by proposition 3.5 and $(37), \bar{x}$ is a primal optimal solution and if $\bar{\mu}$ is a limit point of $\left\{\mu^{k}\right\}$, using (36) and proposition 3.6, $d(\bar{\mu})=\widehat{f}=\widehat{d}$. Hence $\bar{\mu}$ is a dual optimal solution.

\section{Concluding remarks}

For the first time there appear rational powers $p$ in the multiplier vector, this leads us to think about the value of $p$ with the best convergence rate in the context of multiplier methods. It was not treated in this paper and remains as an open problem, although we observe in computational testing that the number of iterations in the main algorithm decrease when $p$ increases but it causes a bad numerical performance.

Multiplier methods without shift with $p=3$ was considered in [4] without a complete convergence rate study but with a similar convergence analysis.

Convergence analysis for the case $\mathrm{p}=1$ can be obtained following similar hypothesis and theorems as in [9]. In [13] convergence results were considered for $p=0$ for a specific penalty function without shift. Other considerations about the value of $p$, for example, $p \leq 0$ remain as open problems.

Finally, we could consider shifts at each iteration as $\theta\left(\tilde{y}_{i}^{k}\right)=c_{i}^{k}$ and to choose $c_{i}^{k}=r^{k} \alpha^{k}$ as in [16] in order to relate both approaches. 


\section{Appendix}

Following definition 2.1 in [9] we next show that the generalized like-distance considered in this work is a divergence measure.

Proposition 5.1. Given $\theta^{*}$ satisfying conditions $\left.\left.1 b\right)-4 b\right), p \geq 0, r \in[0,1]$ and $c>0$ then

$$
d_{\theta^{*}}^{p}(x, y)=\sum_{i=1}^{m}\left[\frac{y_{i}^{p}}{c} \theta^{*}\left(\frac{c x_{i}}{y_{i}}\right)-\frac{y_{i}^{p}}{c} \theta^{*}(c)-y_{i}^{p-1}\left(\theta^{*}\right)^{\prime}(c)\left(x_{i}-y_{i}\right)\right]
$$

is a divergence measure in $R_{++}^{n}$.

Proof.

1) Since $\theta^{*}$ is convex, $\forall z, w \in R \quad \theta^{*}(z) \geq \theta^{*}(w)+\left(\theta^{*}\right)^{\prime}(w)(z-w)$, in particular for $w=c$ and $z=\frac{c x_{i}}{y_{i}}, \quad i=1, \ldots, m$ with $x_{i} \geq 0, y_{i}>0$ and $\operatorname{dom} \theta^{*}=(0,+\infty)$, we have

$$
\theta^{*}\left(\frac{c x_{i}}{y_{i}}\right) \geq \theta^{*}(c)+\left(\theta^{*}\right)^{\prime}(c)\left(\frac{c x_{i}}{y_{i}}-c\right)
$$

and for all $i=1, \ldots, m$ and $p \geq 0$

$$
\frac{y_{i}^{p}}{c} \theta^{*}\left(\frac{c x_{i}}{y_{i}}\right)-\frac{y_{i}^{p}}{c} \theta^{*}(c)-y_{i}^{p-1}\left(\theta^{*}\right)^{\prime}(c)\left(x_{i}-y_{i}\right) \geq 0
$$

SO

$$
\sum_{i=1}^{m}\left[\frac{y_{i}^{p}}{c} \theta^{*}\left(\frac{c x_{i}}{y_{i}}\right)-\frac{y_{i}^{p}}{c} \theta^{*}(c)-y_{i}^{p-1}\left(\theta^{*}\right)^{\prime}(c)\left(x_{i}-y_{i}\right)\right] \geq 0
$$

that is $d_{\theta^{*}}^{p}(x, y) \geq 0$.

2) Let $\left\{x^{k}\right\}$ be a sequence on $(0, \infty)$. We next show

$$
\lim _{k \rightarrow+\infty} d_{\theta^{*}}^{p}\left(x, x^{k}\right)=0 \Leftrightarrow \lim _{k \rightarrow+\infty} x^{k}=x .
$$

Note that,

i) $\theta^{*}$ is non-increasing on $(0, \kappa)$ and increasing on $(\kappa,+\infty)$.

ii) $\left(\theta^{*}\right)^{\prime}(\kappa)=0$.

iii) $\lim _{t \rightarrow+\infty} \theta^{*}(t)=+\infty$.

So,

$\lim _{k \rightarrow+\infty} d_{\theta^{*}}^{p}\left(x, x^{k}\right)=0$

$\Leftrightarrow \lim _{k \rightarrow+\infty} \sum_{i=1}^{m}\left[\frac{\left(x_{i}^{k}\right)^{p}}{c} \theta^{*}\left(\frac{c x_{1}}{x_{i}^{k}}\right)-\frac{\left(x_{i}^{k}\right)^{p}}{c} \theta^{*}(c)-\left(x_{i}^{k}\right)^{p-1}\left(\theta^{*}\right)^{\prime}(c)\left(x_{i}-x_{i}^{k}\right)\right]=0 \Leftrightarrow$

$\lim _{k \rightarrow+\infty}\left[\frac{\left(x_{i}^{k}\right)^{p}}{c} \theta^{*}\left(\frac{c x_{i}}{x_{i}^{k}}\right)-\frac{\left(x_{i}^{k}\right)^{p}}{c} \theta^{*}(c)-\left(x_{i}^{k}\right)^{p-1}\left(\theta^{*}\right)^{\prime}(c)\left(x_{i}-x_{i}^{k}\right)\right]=0$, 
for all $i=1, \ldots, m$

$\Leftrightarrow \lim _{k \rightarrow+\infty} \theta^{*}\left(\frac{c x_{i}}{x_{i}^{k}}\right)=\theta^{*}(c)$, for all $i=1, \ldots, m \Leftrightarrow \lim _{k \rightarrow+\infty} \frac{c x_{i}}{x_{i}^{k}}=c$, for $i=1, \ldots, m$

$\Leftrightarrow \lim _{k \rightarrow+\infty} \frac{x_{i}}{x_{i}^{k}}=1, i=1, \ldots, m$

$\Leftrightarrow \lim _{k \rightarrow+\infty} x_{i}^{k}=x_{i}, i=1, \ldots, m$

$\Leftrightarrow \lim _{k \rightarrow+\infty} x^{k}=x$.

3) The level set $\Gamma_{1}(y, v)=\left\{x \in(0,+\infty) / d_{\theta^{*}}^{p}(x, y) \leq v\right\}$ is bounded for all $y \in(0,+\infty)$ and for all $v \in(0,+\infty)$.

In fact, suppose for some $y \in(0,+\infty)$ and for some $v \in(0,+\infty), \Gamma(y, v)$ is not bounded.

Then there exists $\left\{x^{k}\right\}$ in $(0,+\infty) \quad / \quad x_{k} \rightarrow+\infty$, but $d_{\theta^{*}}^{p}(x, y) \leq v$, from (14),

$0 \leq \sum_{i=1}^{m} \frac{y_{i}^{p}}{c} \theta^{*}\left(\frac{c x_{i}^{k}}{y_{i}}\right)-\frac{y_{i}^{p}}{c} \theta^{*}(c)-y_{i}^{p-1}\left(\theta^{*}\right)^{\prime}(c)\left(x_{i}^{k}-y_{i}\right) \leq v$

and for $\mathrm{i}=1, \ldots \mathrm{m}$

$$
\begin{aligned}
& 0 \leq \frac{y_{i}^{p}}{c} \theta^{*}\left(\frac{c x_{i}^{k}}{y_{i}}\right)-\frac{y_{i}^{p}}{c} \theta^{*}(c)-y_{i}^{p-1}\left(\theta^{*}\right)^{\prime}(c)\left(x_{i}^{k}-y_{i}\right) \leq v, \text { so } \\
& 0 \leq \frac{y_{i}^{p}}{c} \theta^{*}\left(\frac{c x_{i}^{k}}{y_{i}}\right)-y_{i}^{p-1}\left(\theta^{*}\right)^{\prime}(c) x_{i}^{k} \leq v+\frac{y_{i}^{p}}{c} \theta^{*}(c)-y_{i}^{p-1}\left(\theta^{*}\right)^{\prime}(c) y_{i}, \text { and } \\
& 0 \leq \frac{y_{i}^{p}}{c} \theta^{*}\left(\frac{c x_{i}^{k}}{y_{i}}\right)-y_{i}^{p-1}\left(\theta^{*}\right)^{\prime}(c) x_{i}^{k} \leq v+\frac{y_{i}^{p}}{c} \theta^{*}(c)-y_{i}^{p}\left(\theta^{*}\right)^{\prime}(c) .
\end{aligned}
$$

If $M_{i}=v+\frac{y_{i}^{p}}{c} \theta^{*}(c)-y_{i}^{p}\left(\theta^{*}\right)^{\prime}(c)$ for $i=1, \cdots, m$ we have:

$$
\begin{gathered}
0 \leq \frac{y_{i}^{p}}{c} \theta^{*}\left(\frac{c x_{i}^{k}}{y_{i}}\right)-y_{i}^{p-1}\left(\theta^{*}\right)^{\prime}(c) x_{i}^{k} \leq M_{i}, \text { and } \\
0 \leq \frac{y_{i}^{p}}{c}\left(\frac{\theta^{*}\left(\frac{c x_{i}^{k}}{y_{i}}\right)}{x_{i}^{k}}\right) \leq \frac{M_{i}}{x_{i}^{k}}+y_{i}^{p-1}\left(\theta^{*}\right)^{\prime}(c) .
\end{gathered}
$$

On the other hand,

$$
\begin{aligned}
& \lim _{k \rightarrow+\infty} \frac{y_{i}^{p}}{c}\left(\frac{\theta^{*}\left(\frac{c x_{i}^{k}}{y_{i}}\right)}{x_{i}^{k}}\right)=\lim _{k \rightarrow+\infty} \frac{y_{i}^{p}}{c}\left(\frac{\theta^{*}\left(\frac{c x_{i}^{k}}{y_{i}}\right)}{x_{i}^{k}}\right) \\
& =\frac{y_{i}^{p}}{c} \lim _{k \rightarrow+\infty}\left(\theta^{*}\right)^{\prime}\left(\frac{c x_{i}^{k}}{y_{i}}\right) \frac{c}{y_{i}} \\
& =y_{i}^{p-1} \cdot(+\infty)=+\infty .
\end{aligned}
$$

Furthermore : $\frac{M_{i}}{x_{i}^{k}}+y_{i}^{p-1}\left(\theta^{*}\right)^{\prime}(c) \rightarrow y_{i}^{p-1}\left(\theta^{*}\right)^{\prime}(c)$, we obtain $+\infty \leq y_{i}^{p-1}\left(\theta^{*}\right)^{\prime}(c)$, which is a contradiction.

4) Next we prove that $\Gamma_{2}(x, v)=\left\{y \in(0,+\infty) / d_{\theta^{*}}^{p}(x, y) \leq v\right\}$ is bounded 
for all $x \in(0,+\infty)$ and for all $v \in(0,+\infty)$.

Suppose there exists $\left\{y^{k}\right\} \subset \Gamma_{2}(x, v)$ such that for some $j ;\left\{y_{j}^{k}\right\}$ is not bounded and without loss of generality $y_{j}^{k+1}>y_{j}^{k}, \forall k$. For $x$ fixed, since $\frac{x_{j}}{y_{j}^{k}} \rightarrow 0$ if $k \rightarrow+\infty$, then for sufficiently large $k: \frac{x_{j}}{y_{j}^{k}}<1$ implying that $\frac{c x_{j}}{y_{j}^{k}}<c$ and so

$v \geq \frac{\left(y_{j}^{k}\right)^{p}}{c} \theta^{*}\left(\frac{c x_{j}}{y_{j}^{k}}\right)-\frac{\left(y_{j}^{k}\right)^{p}}{c} \theta^{*}(c)-\left(y_{j}^{k}\right)^{p-1}\left(\theta^{*}\right)^{\prime}(c) x_{j}+\left(y_{j}^{k}\right)^{p-1}\left(\theta^{*}\right)^{\prime}(c)\left(y_{j}^{k}\right)$ $\geq \frac{\left(y_{j}^{k}\right)^{p}}{c}\left(\theta^{*}\right)^{\prime}(\xi) c\left[\frac{x_{j}}{y_{j}^{k}}-1\right]-\left(y_{j}^{k}\right)^{p}\left(\theta^{*}\right)^{\prime}(c)\left(\frac{x_{j}}{y_{j}^{k}}\right)+\left(y_{j}^{k}\right)^{p}\left(\theta^{*}\right)^{\prime}(c), \quad \frac{c x_{j}}{y_{j}^{k}}<\xi<c$.

$=\left(y_{j}^{k}\right)^{p}\left(\theta^{*}\right)^{\prime}(\xi)\left[\frac{x_{j}}{y_{j}^{k}}-1\right]-\left(y_{j}^{k}\right)^{p}\left(\theta^{*}\right)^{\prime}(c)\left[\frac{x_{j}}{y_{j}^{k}}-1\right]$

$=\left(y_{j}^{k}\right)^{p}\left[\left(\theta^{*}\right)^{\prime}(\xi)-\left(\theta^{*}\right)^{\prime}(c)\right]\left[\frac{x_{j}}{y_{j}^{k}}-1\right]$

$=\left(y_{j}^{k}\right)^{p}\left(\theta^{*}\right)^{\prime \prime}(\bar{\xi})(\xi-c)\left[\frac{x_{j}}{y_{j}{ }^{k}}-1\right] \rightarrow+\infty \quad(\xi<\bar{\xi}<c)$

which is a contradiction.

Finally $d_{\theta^{*}}^{p}(x, y)$ is a divergence measure.

\section{References}

[1] A. Auslender, M. Teboulle and S. Ben-Tiba, Interior proximal and multiplier methods based on second order homogeneous kernels, Mathematics of Operations Research 24, No. 3, pp. 645-668, 1999.

[2] A.M. Ben Tal and M. Zibulevsky, Penalty barrier multiplier methods for convex programming problems, SIAM Journal on Optimization 7, pp. 447336, 1997.

[3] D.P. Bertsekas , Constrained Optimization and Lagrange Multiplier Methods, Academic Press, New York, 1982.

[4] J. Campos , R.A. Castillo and E. Hernandez, A multiplier method with a third order homogeneous kernel, Technical Report, U.C.L.A, Venezuela, 2005.

[5] R.A. Castillo and C. Gonzaga, Penalidades generalizadas e metodos de lagrangeano aumentado para promamação nao linear, Dsc. these, U.F.R.J, Brasil, 1998.

[6] J. Eckstein , Nonlinear proximal point algoritms using Bregman funtions, with aplications to convex programming, Mathematics of Operations Researchs, 18, pp. 202-226, 1993.

[7] A. Fiacco and G. McCormick, Nonlinear programming, Sequential Unconstrained Minimization Techniques, Classics in applied Mathemathics, SIAM, Philadhelpia, 1990. 
[8] A. Iusem, Metodos de ponto proximal em Optimização, 20 Coloquio Brasileiro de matematica," IMPA, R.J, Brazil, 1995.

[9] A. Iusem , M. Teboulle and B. Svaiter, Entropy-like proximal methods in convex programming, Mathematics of Operations Research, 19(4), pp. 790-814, 1994.

[10] A. Iusem , Augmented Lagrangian methods and proximal point methods for convex optimization, Investigacion Operativa, 8 , pp. 11-50, 1997.

[11] J.B. Hiriart-Urruty and C. Lemarechal, Convex Analysis and Minimization Algorithm II, Springer Verlag, New York, 1996.

[12] K. Kiwiel , Proximal minimization methods with generalized Bregman funtions, SIAM J. on Control and Optimization, 35, 1142-1168, 19(4), 1997.

[13] L. Matioli, Tese de Doutorado, Univesidade Federal de Santa Catarina, Brazil, 2000.

[14] R. Poliak and M. Teboulle, Nonlinear rescaling and proximal-like methods in convex programming, 76, pp. 667-739, 1997.

[15] R.T. Rockafellar, Convex Analysis, Princeton University Press, New Jersey, 1970.

[16] P.J. Silva, J. Eckstein and C. Humes, Rescaling and Stepsize selection in proximal methods using separable generalized distances, SIAM Journal on Optimization 12(1), pp. 238-261, 2001.

[17] M. Teboulle, Entropic proximal mappings with applied to nonlinear programming, Mathematics of Operations Research 17, pp- 97-116, 1992.

\section{Received: November 21, 2006}

